\title{
Explicit Algebraic Coverings of a Pointed Torus
}

Ane S.I. Anema and Jaap Top

\begin{abstract}
This note contains an application of the algebraic study by Schütt and Shioda of the elliptic modular surface attached to the commutator subgroup of the modular group. This is used here to provide algebraic descriptions of certain coverings of a $j$-invariant 0 elliptic curve, unramified except over precisely one point.
\end{abstract}

Key words: Covering, Elliptic surface, Torsion section, Potential stable reduction Mathematics Subject Classifications (2010): Primary 14H30; Secondary 11G05, $14 \mathrm{~J} 27,57 \mathrm{M} 12$

\section{Introduction}

This note was inspired by two recent papers of Jeroen Sijsling [8, 9]. Sijsling finds explicit equations for certain Shimura curves with the special property that they admit a morphism to a curve of genus one; moreover, this morphism is unramified except over precisely one point.

From a topological point of view, the description of such coverings is relatively simple (compare the Introduction of [3]): the fundamental group of a genus one curve minus a point is the free group $F_{2}$ on two generators. So finite coverings correspond to finite index subgroups $H$ of $F_{2}$ and such a covering ramifies over the distinguished point, precisely when the intersection $N=\cap_{g \in F_{2}} g \mathrm{Hg}^{-1}$ of all conjugates of $\mathrm{H}$ in $F_{2}$ satisfies that $F_{2} / N$ is nonabelian.

However, it is far from trivial to find algebraic equations for such a topological covering. In this note we use a particular elliptic surface described by Schütt and Shioda [6] in order to obtain some examples. Namely, they take the elliptic curve

A.S.I. Anema • J. Top $(\varangle)$

JBI-RuG, Nijenborgh 9, 9747 AG Groningen, The Netherlands

e-mail: a.s.i.anema@rug.nl; j.top@rug.nl 
$B / \mathbb{C}$ with $j$-invariant 0 and an elliptic surface $\mathcal{E} \rightarrow B$ which has precisely one singular fibre. Observing that for any integer $n \neq 0$ the $n$-torsion subscheme $\mathcal{E}[n] \rightarrow$ $B$ of $\mathcal{E} \rightarrow B$ is étale except possibly over the point corresponding to the singular fibre, we find coverings of $B$ which are unramified away from this point. In what follows we calculate the ramification indices of such coverings, the degree and hence the genus, and we briefly describe some subcovers.

Most of the results described here were obtained as part of the master's thesis project [1] of the first author in 2011, supervised by the second author. We thank Jeroen Sijsling and Lenny Taelman for their interest in this project.

\section{The Coverings}

Throughout this note we denote by $B / \mathbb{C}$ the elliptic curve corresponding to the affine equation

$$
B / \mathbb{C}: 4 a^{3}+27 b^{2}=1 .
$$

The function field $\mathbb{C}(B)$ of $B / \mathbb{C}$ is the quadratic extension $\mathbb{C}(a, b)$ of the rational function field $\mathbb{C}(a)$ given by $4 a^{3}+27 b^{2}=1$. The unique point of $B$ where the functions $a, b$ have a pole is denoted by $O$. As is well known, $a$ has a pole of order 2 and $b$ has a pole of order 3 at $O$.

The elliptic curve $E / \mathbb{C}(B)$ is defined by the equation

$$
E / \mathbb{C}(B): y^{2}=x^{3}+a x+b .
$$

Then $E / \mathbb{C}(B)$ is the generic fibre of a unique elliptic surface

$$
\mathcal{E} \longrightarrow B
$$

defined over $B$. Up to some scaling factors, this is the elliptic surface studied in [6]. By construction the discriminant of the polynomial $x^{3}+a x+b \in \mathbb{C}(B)[x]$ is a nonzero constant. Hence $\mathcal{E} \rightarrow B$ has smooth fibers over all points in $B(\mathbb{C})$ except the point $O$. It is easy to check that the fiber over $O$ is of type $I_{6}^{*}$ in Kodaira's terminology.

Let $\ell$ be a prime number and denote by $\mathbb{C}(B)(E[\ell])$ the finite extension of $\mathbb{C}(B)$ obtained by adjoining all coordinates of all points on $E$ of order $\ell$. Then $\mathbb{C}(B)(E[\ell])$ is the function field of a unique smooth projective curve $C_{\ell} / \mathbb{C}$. Moreover, the inclusion $\mathbb{C}(B) \subset \mathbb{C}(B)(E[\ell])$ corresponds to a morphism

$$
\pi_{\ell}: C_{\ell} \longrightarrow B
$$

Some properties of these coverings are described in the following result.

Theorem 1. 1. The morphism $\pi_{\ell}: C_{\ell} \rightarrow B$ is Galois and it is unramified away from $O$.

2. The morphism $\pi_{2}$ is unramified of degree 3 .

3. The morphism $\pi_{3}$ has degree 8 with Galois group the quaternion group $\{ \pm 1, \pm i$, $\pm j, \pm k\}$, and its ramification index at every point over $O$ equals 2 . 
4. For $\ell>3$, the Galois group of $\pi_{\ell}$ equals $S L_{2}\left(\mathbb{F}_{\ell}\right)$ and the ramification index at every point over $O$ equals $2 \ell$.

The first claim here is part of the standard results on elliptic curves, see, e.g. [10, VII Proposition 4.1] and [7]. The other assertions will be proven in the next section. Note that the Galois group of $\pi_{\ell}$ is determined by its action on the points of order $\ell$ on the elliptic curve $E$, hence this Galois group is a subgroup of $\mathrm{GL}_{2}(\mathbb{Z} / \ell \mathbb{Z})$. Moreover, since $\mathbb{C}(B)$ contains the $\ell$-th roots of unity, the Galois invariance of the Weil $e_{\ell}$-pairing implies that this Galois group is in fact a subgroup of $\mathrm{SL}_{2}(\mathbb{Z} / \ell \mathbb{Z})$. Theorem 1 above implies in particular which subgroup we have depending on the prime $\ell$ : for $\ell=2$ it is the 3-Sylow subgroup of $\mathrm{SL}_{2}(\mathbb{Z} / 2 \mathbb{Z})$; for $\ell=3$ it is the 2 -Sylow subgroup of $\mathrm{SL}_{2}(\mathbb{Z} / 3 \mathbb{Z})$, and for all primes $\ell>3$ it is the full group $\mathrm{SL}_{2}(\mathbb{Z} / \ell \mathbb{Z})$.

In the last section we briefly discuss some intermediate coverings of the ones described above. These correspond to the intersection of our Galois group with certain subgroups of $\mathrm{SL}_{2}(\mathbb{Z} / \ell \mathbb{Z})$.

\section{The Proofs}

This section contains proofs of the assertions (2), (3) and (4) of Theorem 1.

\subsection{2-Torsion}

See also [7, Sect. 5.3a]. The discriminant of the polynomial $x^{3}+a x+b \in \mathbb{C}(B)[x]$ is a square. Moreover, this cubic polynomial is irreducible: indeed, if $f \in \mathbb{C}(B)$ were a zero, then $f$ would be regular at all points $\neq O$ of $B$ and $f$ would have a pole of order 1 at $O$. Since $B$ has positive genus, such an $f$ does not exist.

It follows that the splitting field over $\mathbb{C}(B)$ of the polynomial $x^{3}+a x+b$ has degree 3. In particular, the Galois group of this splitting field over $\mathbb{C}(B)$ is cyclic of order 3 .

This proves our claims concerning the curves $C_{2} \rightarrow B$ : this map is unramified since it is abelian. An affine curve birational over $\mathbb{C}$ to $C_{2}$ is the curve with coordinate ring

$$
\begin{gathered}
\mathbb{C}[a, b, x] /\left(4 a^{3}+27 b^{2}-1, x^{3}+a x+b\right) \\
\cong \\
\mathbb{C}[a, x] /\left(4 a^{3}+27 x^{6}+54 a x^{4}+27 a^{2} x^{2}-1\right) .
\end{gathered}
$$

The map $(a, x) \mapsto\left(a,-x^{3}-a x\right)$ from the curve with equation $4 a^{3}+27 x^{6}+54 a x^{4}+$ $27 a^{2} x^{2}-1=0$ to $B$ corresponds to the covering $C_{2} \rightarrow B$. 


\subsection{3-Torsion}

See also [7, 5.3b] for a discussion of point of order 3. In particular, since the discriminant of the elliptic curve $E$ is a cube in $\mathbb{C}(B)$, the Galois group of $\pi_{3}: C_{3} \rightarrow B$ is contained in the (unique) 2-Sylow subgroup of $\mathrm{SL}_{2}(\mathbb{Z} / 3 \mathbb{Z})$, which is isomorphic to the quaternion group of order 8 .

Moreover, we claim that $C_{3} \rightarrow B$ is ramified: indeed, recall that the elliptic curve $E$ has additive reduction at the valuation corresponding to $O \in B$. Over $\mathbb{C}\left(C_{3}\right)$, the curve $E$ cannot have additive reduction at any valuation. This is well-known; the argument runs as follows. Suppose $K$ is the completion at a place $v$ where $E$ has additive reduction, and $E(K)$ contains all points of order $n \geq 3$. Since reduction modulo $v$ :

$$
E_{0}(K)[n] \longrightarrow \bar{E}_{\mathrm{ns}}(\mathbb{C}) \cong(\mathbb{C},+)
$$

is injective on torsion points, additive reduction implies that $E_{0}(K)$ has trivial $n$-torsion. Hence $E(K) / E_{0}(K)$ contains a subgroup $\mathbb{Z} / n \mathbb{Z} \times \mathbb{Z} / n \mathbb{Z}$, contradicting the possible structures of this component group.

Since in the extension $\mathbb{C}\left(C_{3}\right) \supset \mathbb{C}(B)$ the reduction of $E$ at (points over) $O$ changes from additive to semi-stable, $C_{3} \rightarrow B$ is ramified over $O$. It is unramified over all other points of $B$, hence we conclude that the Galois group of $C_{3} \rightarrow B$ is not abelian.

Observing that all proper subgroups of the 2-Sylow subgroup of $\mathrm{SL}_{2}(\mathbb{Z} / 3 \mathbb{Z})$ are abelian it follows that the Galois group of $C_{3} \rightarrow B$ equals this 2-Sylow subgroup.

The $x$-coordinates of all points of order 3 on $E$ generate the splitting field of

$$
3 x^{4}+6 a x^{2}+12 b x-a^{2}
$$

over $\mathbb{C}(B)$. This splitting field is, by construction, unramified over all points $\neq O$ of $B$. It is unramified over $O$ as well: indeed, by its construction, the splitting field has as Galois group over $\mathbb{C}(B)$ the image of $\operatorname{Gal}(\mathbb{C}(B)(E[3]) / \mathbb{C}(B))$ under the canonical map

$$
\mathrm{SL}_{2}(\mathbb{Z} / 3 \mathbb{Z}) \longrightarrow \mathrm{PSL}_{2}(\mathbb{Z} / 3 \mathbb{Z})
$$

Under this map, the image of the 2 -Sylow subgroup of $\mathrm{SL}_{2}(\mathbb{Z} / 3 \mathbb{Z})$ is $\cong \mathbb{Z} / 2 \mathbb{Z} \times \mathbb{Z} / 2 \mathbb{Z}$. This implies that the corresponding extension is unramified. Using

$$
C_{3} \longrightarrow C_{3} /( \pm 1) \longrightarrow B
$$

in which the first map is cyclic of degree 2 and the second map is Galois and unramified, with Galois group $\mathbb{Z} / 2 \mathbb{Z} \times \mathbb{Z} / 2 \mathbb{Z}$, it follows that every ramified point of $\pi_{3}$ has ramification index 2 .

This proves the assertions concerning $\pi_{3}$. An alternative, more computational proof of the assertion concerning the ramification index is presented in [1, Proposition 4.4]. 


\section{$3.3 \ell$-Torsion with $\ell \geq 5$}

Now fix a prime $\ell \geq 5$ and consider the extension $\mathbb{C}\left(C_{\ell}\right)=\mathbb{C}(B)(E[\ell]) \supset \mathbb{C}(B)$. To prove the claims presented in Theorem 1 for this case, we recall a result of Igusa [2]; see also [5, Theorem 1].

Define

$$
E^{\prime}: y^{2}=x^{3}-\frac{27 t}{t-1728} x-\frac{54 t}{t-1728} .
$$

This is an elliptic curve over $\mathbb{C}(t)$ with $j\left(E^{\prime}\right)=t$. The result of Igusa referred to above is

Proposition 1. (Igusa) The extension $\mathbb{C}(t)\left(E^{\prime}[\ell]\right) / \mathbb{C}(t)$ is Galois with group $\mathrm{SL}_{2}(\mathbb{Z} / \ell \mathbb{Z})$.

To relate the curve $E^{\prime} / \mathbb{C}(t)$ to the curve $E / \mathbb{C}(a, b)$, note that $j(E)=6912 a^{3}$. Hence identifying $t$ with $j(E)$, which corresponds to the map $B \rightarrow \mathbb{P}^{1}$ given by $(a, b) \mapsto 6912 a^{3}$, the curves $E$ and $E^{\prime}$ are both defined over $\mathbb{C}(B)$ and moreover they have the same $j$-invariant. In fact,

$$
-\frac{27 \cdot 6912 a^{3}}{6912 a^{3}-1728}=-\frac{27 \cdot 4 a^{3}}{4 a^{3}-1}=\left(\frac{2 a}{b}\right)^{2} a
$$

and

$$
-\frac{54 \cdot 6912 a^{3}}{6912 a^{3}-1728}=-\frac{54 \cdot 4 a^{3}}{4 a^{3}-1}=\left(\frac{2 a}{b}\right)^{3} b
$$

This implies that over the quadratic extension $\mathbb{C}(a, b, c)$ of $\mathbb{C}(B)$ defined by $c^{2}=2 a / b$, the curves $E$ and $E^{\prime}$ are isomorphic. Therefore

$$
\mathbb{C}(a, b, c, E[\ell])=\mathbb{C}\left(a, b, c, E^{\prime}[\ell]\right) .
$$

Consider the following diagram of field extensions:

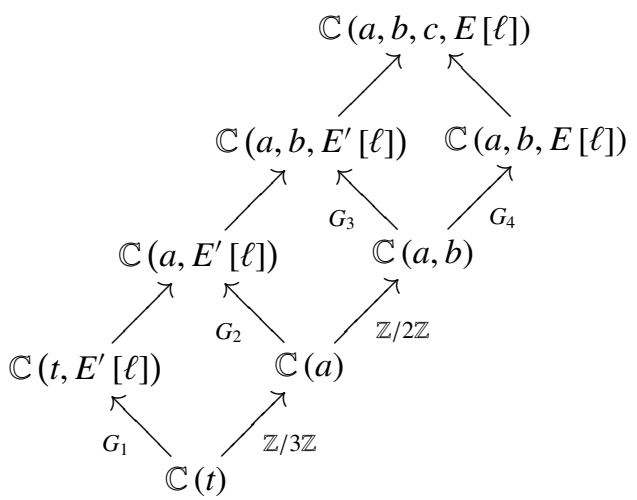


Here the arrows denote Galois extensions, and the corresponding Galois group is written below most of them. The groups $G_{i}$ may be regarded as subgroups of $\mathrm{SL}_{2}(\mathbb{Z} / \ell \mathbb{Z})$. We will study these groups.

By Proposition 1 we have $G_{1}=\mathrm{SL}_{2}(\mathbb{Z} / \ell \mathbb{Z})$. The extension $\mathbb{C}\left(a, E^{\prime}[\ell]\right)$ of $\mathbb{C}\left(t, E^{\prime}[\ell]\right)$ cannot have degree one, since this would imply that $G_{1}=\mathrm{SL}_{2}(\mathbb{Z} / \ell \mathbb{Z})$ would have a normal subgroup $G_{2}$ of index 3 . This is not the case when $\ell>3$ is prime, as is a well-known and classical fact; a recently published (standard) proof can be obtained from [4]. Hence the given extension has degree 3, implying that $G_{2}=\mathrm{SL}_{2}(\mathbb{Z} / \ell \mathbb{Z})$. A similar reasoning $\left(G_{2}\right.$ does not have a subgroup of index 2 for $\ell \neq 2$ ) shows that $G_{3}=\mathrm{SL}_{2}(\mathbb{Z} / \ell \mathbb{Z})$.

Now if $G_{4}$ would not be the full group $\mathrm{SL}_{2}(\mathbb{Z} / \ell \mathbb{Z})$, then

$$
\left[\mathbb{C}(a, b, c, E[\ell]): \mathbb{C}\left(a, b, E^{\prime}[\ell]\right)\right]<[\mathbb{C}(a, b, c, E[\ell]): \mathbb{C}(a, b, E[\ell])] \leq 2,
$$

implying that $\mathbb{C}(a, b, c, E[\ell])=\mathbb{C}\left(a, b, E^{\prime}[\ell]\right)$. The conclusion is that $G_{4}$ would be a subgroup of $\mathrm{SL}_{2}(\mathbb{Z} / \ell \mathbb{Z})$ of index 2 , which is not true since $\ell \geq 5$. This shows that indeed $\mathbb{C}(a, b, E[\ell]) / \mathbb{C}(a, b)$ has Galois group $G_{4}=\mathrm{SL}_{2}(\mathbb{Z} / \ell \mathbb{Z})$, which is one of the assertions in Theorem 1 .

It remains to prove that for $\ell \geq 5$ the map $\pi_{\ell}: C_{\ell} \rightarrow B$ is ramified at every point over $O \in B$ with ramification index $2 \ell$.

A uniformizer at $O$ is $\pi:=2 a / b \in \mathbb{C}(B)$. We consider the curve $E$ over the completion $\mathbb{C}((\pi))$. Note that

$$
a \pi^{2}=-27+b^{-2} \in-27+\pi^{6} \mathbb{C}[[\pi]]
$$

and

$$
b \pi^{3}=-54+2 b^{-2} \in-54+\pi^{6} \mathbb{C}[[\pi]] .
$$

Hence using $c^{2}=\pi=2 a / b$ (which defines the quadratic covering of $B$ used above, which ramifies over $O$ since $c$ is a uniformizer at the point over $O$ ), one finds that over $\mathbb{C}((c))$ the curve $E$ is given as

$$
y^{2}=x^{3}+c^{-4}\left(-27+b^{-2}\right) x+c^{-6}\left(-54+2 b^{-2}\right) .
$$

This is equivalent to

$$
y^{2}=x^{3}+\left(-27+b^{-2}\right) x-54+2 b^{-2}
$$

Modulo (c) the reduction of the above model is

$$
y^{2}=x^{3}-27 x-54=(x+3)^{2}(x-6) .
$$

So we have an elliptic curve with multiplicative reduction over a local field with an algebraically closed residue field. By the theory of the Tate curve as, e.g., explained in [11, Chap. V Theorems 3.1 and 5.3], $q \in \mathbb{C}((c))$ exists such that for every finite extension $L$ of $\mathbb{C}((c))$ we have a $\operatorname{Gal}(L / \mathbb{C}((c)))$-equivariant isomorphism 


$$
L^{*} / q^{\mathbb{Z}} \stackrel{\cong}{\longrightarrow} E(L)
$$

Moreover, the valuation of $q$ equals the valuation of the discriminant of a minimal model of $E$. Such a model is given above, and the valuation of the discriminant is 12 .

Since $\mathbb{C}((c))(E[\ell])=\mathbb{C}((c))(\sqrt[\ell]{q})$ and $q \in c^{12} \mathbb{C}[[c]]^{*}$ and $\ell \geq 5$, one concludes that $\mathbb{C}((c))(E[\ell])=\mathbb{C}((\sqrt[\ell]{c})) \supset \mathbb{C}((c))$ which is a totally ramified extension of degree $\ell$. This yields the extensions

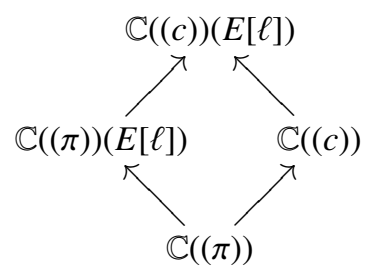

Now observe that any finite extension of $\mathbb{C}((\pi))$ over which the curve $E$ has multiplicative reduction, must contain the element $c$. In particular, the fact that $E$ has all its points of order $\ell$ rational over $\mathbb{C}((\pi))(E[\ell])$ implies that $E$ has multiplicative reduction over this field, hence

$$
c \in \mathbb{C}((\pi))(E[\ell]) .
$$

So this shows that

$$
\mathbb{C}((\pi)) \subset \mathbb{C}((c)) \subset \mathbb{C}((c))(E[\ell])=\mathbb{C}((\pi))(E[\ell]),
$$

in which the first extension is ramified of degree 2 and the second one is ramified of degree $\ell$. As a consequence, the extension $\mathbb{C}((\pi))(E[\ell]) / \mathbb{C}((\pi))$ is ramified of degree $2 \ell$.

This finishes the proof of Theorem 1. As a remark, one may prove part of the above result by combining a topological and a group theoretic argument. Namely, the topology of the torus implies that the inertia group at any ramified point over $O$ is generated by a commutator $A B A^{-1} B^{-1}$ with $A, B \in \mathrm{SL}_{2}(\mathbb{Z} / \ell \mathbb{Z})$. Using some group theory one shows that such a commutator cannot have order $\ell$, hence since its order is divisible by $\ell$, it necessarily equals $2 \ell$.

Using that $\# \mathrm{SL}_{2}(\mathbb{Z} / \ell \mathbb{Z})=\ell^{3}-\ell$ it is now straightforward to compute the genus of the curves $C_{\ell}$. One finds

$$
\begin{aligned}
& g\left(C_{2}\right)=1, \\
& g\left(C_{3}\right)=3,
\end{aligned}
$$

and

$$
g\left(C_{\ell}\right)=1+\left(\ell^{2}-1\right)(2 \ell-1) / 4
$$

for any prime number $\ell \geq 5$. 


\section{Intermediate Coverings}

Rather than adjoining the coordinates of all points of order $\ell$ on $E$ to the field $\mathbb{C}(B)$, one can adjoin only the $x$-coordinates of these points, or only the coordinates of one point, or only the $x$-coordinate of one point. This results in an intermediate field between $\mathbb{C}(B)$ and $\mathbb{C}\left(C_{\ell}\right)$, hence with a subgroup of the corresponding Galois group. We briefly discuss this here.

\subsection{All $x$-Coordinates}

Let $\mathbb{C}\left(D_{\ell}\right)$ be the subfield of $\mathbb{C}\left(C_{\ell}\right)$ obtained by adjoining all $x$-coordinates of the points of order $\ell$ on $E$, to the field $\mathbb{C}(B)$. This extension corresponds to coverings of curves

$$
C_{\ell} \longrightarrow D_{\ell} \longrightarrow B
$$

and to the (normal) subgroup

$$
\{ \pm 1\} \cap \operatorname{Gal}\left(\mathbb{C}\left(C_{\ell}\right) / \mathbb{C}(B)\right),
$$

Hence it is Galois over $\mathbb{C}(B)$, with Galois group equal to the image of $\operatorname{Gal}\left(\mathbb{C}\left(C_{\ell}\right)\right.$ / $\mathbb{C}(B))$ under the canonical map

$$
\mathrm{SL}_{2}(\mathbb{Z} / \ell \mathbb{Z}) \longrightarrow \mathrm{PSL}_{2}(\mathbb{Z} / \ell \mathbb{Z})
$$

For $\ell=2$ this canonical map is a bijection and $\mathbb{C}\left(D_{\ell}\right)=\mathbb{C}\left(C_{\ell}\right)$ (as is obvious since the $y$-coordinates of points of order 2 on $E$ are zero).

For $\ell=3$ the image is, as was already discussed earlier, the group $\mathbb{Z} / 2 \mathbb{Z} \times \mathbb{Z} / 2 \mathbb{Z}$. A consequence of this is that the polynomial

$$
3 x^{4}+6 a x^{2}+12 b x-a^{2}
$$

is irreducible over $\mathbb{C}(B)$ : indeed, this polynomial does not have a zero in $\mathbb{C}(B)$, since a root would be a function with a pole of order one at $O$ and no other poles; such a function does not exist.

If the bi-quadratic polynomial would factor as a product of two quadratics $f_{1}$ and $f_{2}$, then in particular its discriminant (which is a nonzero constant) would equal the product of the discriminants of $f_{1}$ and $f_{2}$ times the square of the resultant of $f_{1}$ and $f_{2}$. Hence $f_{1}$ and $f_{2}$ would have the same splitting field, contradicting the fact that the bi-quadratic polynomial has a Galois group of order 4 .

Finally, for $\ell \geq 5$ one has that $D_{\ell} \rightarrow B$ is Galois with group $\operatorname{PSL}_{2}(\mathbb{Z} / \ell \mathbb{Z})$. The image in this group of a cyclic subgroup in $\mathrm{SL}_{2}(\mathbb{Z} / \ell \mathbb{Z})$ of order $2 \ell$, has order $\ell$. As a result, $D_{\ell} \rightarrow B$ ramifies over $O \in B$ with ramification index $\ell$.

For the genera of the curves $D_{\ell}$, these observations imply 


$$
g\left(D_{2}\right)=1=g\left(D_{3}\right)
$$

and

$$
g\left(D_{\ell}\right)=1+\left(\ell^{2}-1\right)(\ell-1) / 4
$$

for all primes $\ell \geq 5$.

\subsection{One Point}

Fix a point $P \in E$ of exact $\operatorname{order} \ell$. This yields a tower of extensions

$$
\mathbb{C}(B) \subset \mathbb{C}(B)(P) \subset \mathbb{C}\left(C_{\ell}\right)
$$

in which the last extension is Galois with group $\left(\begin{array}{ll}1 & * \\ 0 & 1\end{array}\right)$ of order $\ell$ (provided $\ell \geq 5$, otherwise one has $\mathbb{C}(B)(P)=\mathbb{C}\left(C_{\ell}\right)$.) In particular, for $\ell \geq 5$ it follows that the ramification index over $O$ in any point of the first extension is one of 2 or $2 \ell$. Moreover, in this case the inertia group at a point of $C_{\ell}$ over $O \in B$ is a cyclic subgroup of order $2 \ell$ in $\mathrm{SL}_{2}(\mathbb{Z} / \ell \mathbb{Z})$. The group $\mathrm{SL}_{2}$ permutes these ramified points in $C_{\ell}$, and this yields the conjugation action of $\mathrm{SL}_{2}$ on the set of cyclic subgroups of order $2 \ell$. There are precisely $\ell+1$ such subgroups (corresponding to the one-dimensional subspaces of $\left.\mathbb{F}_{\ell}^{2}\right)$. The number of ramified points in $C_{\ell} \rightarrow B$ over $O$ equals $\left(\ell^{3}-\ell\right) /(2 \ell)$. Hence each of the subgroups of order $2 \ell$ appears $(\ell-1) / 2$ times as inertia group of a point in $C_{\ell} \rightarrow B$.

It follows that in $\mathbb{C}(B) \subset \mathbb{C}(B)(P)$ we have $(\ell-1) / 2$ points with ramification degree 2 (corresponding to the points in $C_{\ell}$ which have $\pm\left(\begin{array}{ll}1 & * \\ 0 & 1\end{array}\right)$ as inertia group), and $(\ell-1) / 2$ points with ramification index $2 \ell$ (corresponding to the remaining ramification points in $C_{\ell} \rightarrow B$ ).

As a consequence, the genus of the corresponding covering of $B$ equals $1+\ell$ $(\ell-1) / 2$. Note that for $\ell=2$ and for $\ell=3$ this genus is respectively 1 and 3 , as was shown earlier.

\subsection{One $x$-Coordinate}

In a similar manner one may treat the extension

$$
\mathbb{C}(B) \subset \mathbb{C}(B)(x(P))
$$

obtained by adjoining the $x$-coordinate of one point of exact order $\ell$.

For $\ell \leq 3$ this yields an unramified extension, hence a curve of genus one (these curves were already described above).

For $\ell \geq 5$ a prime number, the extension degree equals $\left(\ell^{2}-1\right) / 2$ and over $O$ one finds $(\ell-1) / 2$ unramified points and $(\ell-1) / 2$ points with ramification index $\ell$. Consequently, the corresponding genus equals $1+(\ell-1)^{2} / 4$. 


\section{References}

1. A.S.I. Anema, Branched covering spaces of an elliptic curve that branch only above a single point, Master's thesis, Groningen, (2011) http://irs.ub.rug.nl/dbi/4e707a67dac82. Accessed 20 July 2012

2. J.-I. Igusa, Fibre systems of Jacobian varieties: (III. Fibre systems of elliptic curves). Am. J. Math. 81, 453-476 (1959)

3. H.W. Lenstra, Galois theory for schemes. Lecture Notes (2008) http://websites.math. leidenuniv.nl/algebra/GSchemes.pdf. Accessed on 20 July (2012)

4. J. Maciel, $\mathrm{SL}_{n}(F)$ equals its own derived group. Int. J. Algebra 2, 585-594 (2008)

5. D.E. Rohrlich, Modular curves, Hecke correspondences, and $L$-functions, in Modular Forms and Fermat's Last Theorem, ed. by G.Cornell, J.H. Silverman, G. Stevens (Springer, New York, 1997), pp. 41-100

6. M. Schütt, T. Shioda, An interesting elliptic surface over an elliptic curve. Proc. Jpn. Acad. Sci. 83, 40-45 (2007)

7. J.-P. Serre, Propriétés galoisiennes des points d'ordre fini des courbes elliptiques. Invent. Math. 15, 259-331 (1972)

8. J. Sijsling, Arithmetic (1; e)-curves and Belyĭ maps. Math. Comp. 81, 1823-1855 (2012)

9. J. Sijsling, Canonical models of arithmetic $(1 ; e)$-curves. Math. Zeitschrift 271, 38 (2012)

10. J.H. Silverman, The Arithmetic of Elliptic Curves. Graduate Texts in Mathematics, vol. 106 (Springer, New York, 1986)

11. J.H. Silverman, Advanced Topics in the Arithmetic of Elliptic Curves. Graduate Texts in Mathematics, vol. 151 (Springer, New York, 1994) 